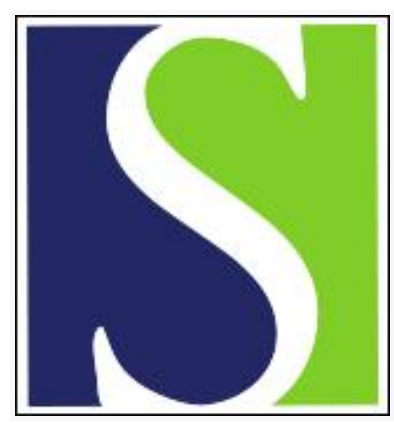

Scand J Work Environ Health 2003;29(1):22-26

https://doi.org/10.5271/sjweh.700

Issue date: Feb 2003

Timed bright-light exposure and complaints related to shift work among women

by Leppämäki S, Partonen T, Piiroinen P, Haukka J, Lönnqvist J

Affiliation: National Public Health Institute, Department of Mental Health, Mannerheimintie 166, Fl-00300 Helsinki, Finland. sami.leppamaki@ktl.fi

Refers to the following texts of the Journal: 1998;24 suppl 3:115-120 1999;25(6):610-615 1996;22(2):133-138

Key terms: bright-light exposure; circadian phase; phototherapy; seasonality; shift work; woman

This article in PubMed: www.ncbi.nlm.nih.gov/pubmed/12630432

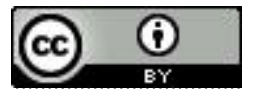




\title{
Timed bright-light exposure and complaints related to shift work among women
}

\author{
by Sami Leppämäki, MD, ${ }^{1}$ Timo Partonen, MD, ${ }^{1}$ Pauliina Piiroinen, MD, ${ }^{2}$ Jari Haukka, PhD, ${ }^{1}$ Jouko \\ Lönnqvist, MD ${ }^{1}$
}

Leppämäki S, Partonen T, Piiroinen P, Haukka J, Lönnqvist J. Timed bright-light exposure and complaints related to shift work among women. Scand J Work Environ Health 2003;29(1):22-26.

\begin{abstract}
Objectives This field study measured whether repeated, brief exposures to bright light during night shifts improved subjective well-being during and after night work. A secondary objective was to investigate whether this response differed by season (summer or winter), seasonality, or age.

Methods Eighty-seven healthy female nurses were voluntarily exposed to brief periods $(4 \times 20$ minutes $)$ of bright (5000 lux) light at scheduled times during every night shift over a 2 -week period. Each morning following a night shift the subjects filled out self-assessment questionnaires measuring subjective symptoms and distress caused by work at night. The questionnaires were also completed 2 weeks before and after the light intervention. The study had two phases, summer (May-June) and winter (November-December). Thirty-seven of the subjects participated during both periods.

Results Light significantly alleviated the subjective distress associated with nightshift work, both in summer and in winter, independent of the subject's age. The effect was stronger for those who reported routine seasonal changes in mood.

Conclusions Short pulses of timed bright-light exposure may enhance subjective adaptation to night work.
\end{abstract}

Key terms circadian phase, phototherapy, seasonality.

Night work conflicts with the internal circadian clock, and shift work schedules have been proposed that would interfere with circadian rhythms as little as possible (1). Light is the strongest known time-giver of the inner clock, and artificial bright or medium-intensity light has been used to shift the phase of circadian rhythms to achieve a faster adaptation to night work (2). Bright light (over 2500 lux) is the treatment of choice for seasonal affective disorder in winter (3), and recent studies indicate that light may have a positive effect on mood even in the absence of depressive disorder (4). A previous study found short (20 minutes) periods of bright light during night shifts to be beneficial in terms of reduced tiredness and higher performance (5). This finding has important practical implications because, in most workplaces (eg, hospital wards), the 4-hour to all- night bright-light conditions used in simulated nightshift studies are not feasible. Using a larger population and a real-time field setting, our study aimed at determining (i) whether brief, timed bright-light pulses reduce subjective distress related to night work, (ii) if the response varies according to season (summer versus winter), and (iii) the factors predicting a person's response to this intervention.

\section{Subjects and methods}

The subjects were nurses currently working on night shifts. Head nurses of wards in four central hospitals in southern Finland were informed of the study protocol

1 National Public Health Institute, Department of Mental Health and Alcohol Research, Helsinki, Finland.

2 University of Helsinki, Department of Psychiatry, Helsinki, Finland.

Reprint requests to: Dr S Leppämäki, National Public Health Institute, Department of Mental Health, Mannerheimintie 166, FIN-00300 Helsinki, Finland. [E-mail: sami.leppamaki@ktl.fi] 
and provided with information leaflets to be delivered to nurses who volunteered for the study. Portable light fixtures with two 55-watt, cool white, fluorescent lamps (Philips Ltd, Eindhoven, The Netherlands) emitting a light intensity of 5000 lux at eye-level were delivered to the wards. The subjects were instructed to use the devices for 20 minutes, between 2200 and 2300, 2400 and 0100,0200 and 0300 , and 0400 and 0500 , as their duties allowed. The study was carried out in 1999 during the summer (10 May to 20 June) and winter (8 November to 19 December). The length of daylight on these dates was 16 hours, 45 minutes to 18 hours, 56 minutes and 8 hours, 7 minutes to 5 hours, 50 minutes, respectively. Each 6-week period was divided into baseline, light exposure, and follow-up periods of 2 weeks.

\section{Assessment}

Subjective evaluations of nightwork-induced symptoms were recorded after each night shift using the Scale for Shift-Work Complaints (SSC), a 17-item questionnaire adopted from the Columbia Jet Lag Scale (6) (appendix). Before the study, all the subjects completed the Seasonal Pattern Assessment Questionnaire (SPAQ) (7), a 26-item abbreviated version of FINRISK (8) and the RAND 36-item Health Survey (RAND) (9). The subjects who participated in the winter also filled out the Horne-Ostberg Morningness-Eveningness Questionnaire (MEQ) (10). The SPAQ measures seasonal changes in mood and behavior and includes a 6-item scale giving the Global Seasonality Score (GSS). The SPAQ criteria for subsyndromal seasonal affective disorder described by Bartko \& Kasper (11) were used as a basis for assessing seasonal changes in mood for each subject. This variable was named seasonality, with values "yes" or "no". The RAND measures eight dimensions of functioning that reflect the perceived health-related quality of life: physical and social functioning, general health, limitations due to physical health or emotional problems, pain, vitality, and emotional well-being. The dimensions used in the RAND are identical to those in the Medical Outcomes Study 36-item Short Form Health Survey (SF-36) (12), but the scoring algorithm used is somewhat different. The abbreviated FINRISK consists of 26 questions concerning smoking, alcohol consumption, dietary fat intake, and habitual exercise.

\section{Ethics}

The study protocol was approved by the Ethics Committee of the National Public Health Institution. Written informed consent was obtained from the subjects before their participation.

\section{Subjects}

One hundred and four women enrolled for the study and returned the baseline questionnaires. Their mean age was 38.8 (SD 7.9, range 22-57) years, and their mean body mass index was 24.0 (SD 3.7 ) $\mathrm{kg} / \mathrm{m}^{2}$. Thirty-two $(31 \%)$ of the nurses reported having at least a subsyndromal degree of seasonal difficulties as measured with the SPAQ (11). Fifty-three (51\%) had smoked, although only $13(12.5 \%)$ did so during the study period, and 67 (64\%) reported consuming alcohol only occasionally or not at all. Fifty-three (51\%) exercised regularly at least twice a week. There were 85 subjects in the summer and 56 in the winter, 37 of whom enrolled for both study periods. All were working on a rotating shift schedule, with one to seven night shifts in each of the 2-week periods. The night shifts started between 2100 and 2200 and ended usually at 0700 .

\section{Statistics}

The main outcome measure was the absolute score on the SSC, recorded after each night shift. To estimate the intervention effect with the SSC, a linear mixed-effects model for longitudinal data was used (13). The model accounts for the intraindividual correlation between measurements by setting the variation as a random effect and treating each person as a cluster in the modeling. In the model, the SSC was set as the dependent variable, and light exposure, baseline characteristics, and their interactions were the independent fixed effects (covariates). For the estimates, 95\% confidence intervals $(95 \% \mathrm{CI})$ were calculated, and t-tests (significance level 0.05 ) were performed on the hypothesis that the estimate differed from zero. The data were screened and analyzed with the S-Plus 2000 Professional Edition for Windows, Release 1 (Mathsoft Inc).

\section{Results}

Of the 104 subjects who returned the baseline questionnaires, $86(83 \%)$ participated in the study, $59(69 \%)$ in the summer and $52(60 \%)$ in the winter. The subjects who entered the study did not differ from those who did not with respect to any of the baseline characteristics. The dropouts were mainly due to incongruity between the work schedules and the study periods (no night shifts, holidays). Thus the final analysis comprised data from 34,27 , and 25 women who participated only in the summer, winter, and both periods, respectively. The mean age was 39.2 (SD 7.8, range 22-57) years, and the mean body mass index was 24.0 (SD 3.5, range 
Table 1. Estimates of the effects (linear mixed-effects model) of light, time of year, seasonal changes in mood (seasonality), and their interactions on the Scale for Shift-work Complaints. ${ }^{\text {a }}(95 \%$ $\mathrm{Cl}=95 \%$ confidence interval, NS=not significant)

\begin{tabular}{lrrl}
\hline & Estimate & \multicolumn{1}{c}{$95 \% \mathrm{Cl}$} & P-value \\
\cline { 2 - 5 } Use of light versus no light & -4.3 & $-7.9--0.7$ & 0.02 \\
Seasonality (yes versus no) & 6.0 & $1.0-11.0$ & 0.02 \\
Time of year (summer versus winter) & -1.1 & $-4.6-2.4$ & NS \\
Light $\times$ seasonality & -5.6 & $-9.7--1.4$ & 0.01 \\
Time of year $\times$ seasonality & -1.0 & $-6.8-4.8$ & NS \\
Time of year $\times$ light & 0.0 & $-4.0-4.0$ & NS
\end{tabular}

a Number of observations 492, number of subjects 86 .

Table 2. Score on the Scale for Shift-Work Complaints during the study periods according to seasonality.

\begin{tabular}{|c|c|c|c|c|c|c|}
\hline & \multicolumn{2}{|c|}{ Baseline period } & \multicolumn{2}{|c|}{ Light exposure } & \multicolumn{2}{|c|}{ Follow-up } \\
\hline & Mean & SD & Mean & SD & Mean & SD \\
\hline No seasonality & 21.0 & 9.9 & 16.2 & 9.9 & 17.1 & 10.2 \\
\hline Seasonality & 25.0 & 8.6 & 13.2 & 5.7 & 25.8 & 14.1 \\
\hline All subjects & 23.6 & 9.6 & 17.8 & 9.2 & 19.6 & 11.3 \\
\hline
\end{tabular}

Table 3. Estimates of the effect of the baseline characteristics on the Scale for Shift-work Complaints. ${ }^{a}(95 \% \mathrm{Cl}=95 \%$ confidence interval, NS=not significant)

\begin{tabular}{lccl}
\hline & Estimate & $95 \% \mathrm{Cl}$ & P-value \\
\cline { 2 - 4 } & & $-1.9-5.6$ & $\mathrm{NS}$ \\
Age ( $\geq 40$ years versus $<40$ years) & 1.9 & $-2.7-7.3$ & $\mathrm{NS}$ \\
Body mass index ( $\geq 25$ versus $<25)$ & 2.3 & $-2.6-4.6$ & $\mathrm{NS}$ \\
Regular exercise versus exercise & 1.0 & -4.5 & NS \\
not at all or seldom & & & \\
Previous high cholesterol (yes versus no) & -0.2 & $-4.8-4.5$ & \\
Smoking (yes versus no) & 3.6 & $-3.7-10.8$ & NS \\
Consumption of alcoholic beverages & & & \\
(seldom to often versus not at all & 0.7 & $-3.0-4.4$ & NS \\
or occasionally) & & & \\
RAND scales (see reference 9) & & & \\
$\quad$ Physical functioning & -0.1 & $-0.4-0.2$ & NS \\
Limitations due to physical health & -0.03 & $-0.09-0.03$ & NS \\
Limitations due to emotional & -0.08 & $-0.1--0.03$ & 0.001 \\
problems & & & \\
Vitality & -0.1 & $-0.2--0.03$ & 0.01 \\
Emotional well-being & -0.2 & $-0.3--0.05$ & 0.006 \\
Social functioning & -0.1 & $-0.2--0.05$ & 0.001 \\
Pain & -0.01 & $-0.1-0.08$ & NS \\
General health & -0.07 & $-0.2-0.05$ & NS \\
\hline
\end{tabular}

19-39) $\mathrm{kg} / \mathrm{m}^{2}$. Twenty-six (30\%) reported having experienced seasonal changes in mood and behavior. The mean number of night shifts during the baseline and light periods was 3.0 (SD 1.8) and 2.9 (SD 1.7), respectively.

The mean SSC score after the first night shift was 24.6 (95\% CI 22.4-26.8) overall, 25.1 (95\% CI 21.928.4) in the summer, 24.4 (95\% CI 21.3-27.4) in the winter, and 28.3 (95\% CI 24.7-32.0) versus 23.2 (95\% CI 20.5 to 26.0) for the subjects reporting seasonal symptoms versus no such changes. Bright-light intervention reduced the scores on the SSC during the study, while the seasonality trait had the opposite effect (table 1). The light $\times$ seasonality interaction was significant $(\mathrm{P}=0.01)$, the reduction in the symptom score being more pronounced for the subjects with the seasonality trait (mean $-9.9,95 \% \mathrm{CI}-13.0--6.7$, contrast: no light and no seasonality). Table 2 shows the mean values of the SSC during the study periods. Season (summer or winter) was taken into account, but it did not influence the results. When mixed-effects models with light exposure, seasonality, and time of year as random effects were tested against the fixed-effects models (analysis of variance), they did not add to the power of the statistical analysis. Table 3 presents the effects of the remaining baseline characteristics of the SSC.

Ten subjects reported side effects possibly related to bright-light exposure: headache ( 8 subjects, 6 reporting concomitant nausea) and irritation of the eyes ( 2 subjects).

\section{Discussion}

Our main finding was that short pulses of bright light appeared to improve subjective well-being during and after the night shifts of our subjects. Similar findings have been reported by Costa et al (5), who also used short periods $(4 \times 20 \mathrm{~min})$ of moderately bright $(2350$ lux) light exposure, and by Iwata et al (14) with a total of 30 minutes of light (over 3000 lux) exposure per shift. Four hours of light increased subjective alertness in a study by Foret et al (15), although the light used was not bright (700-1000 lux), whereas, in a similar design using bright (over 4000 lux) light, no effect on alertness and performance was found in a group of middleaged subjects (16).

To our knowledge, the effect of seasonality on subjective adaptation to shift work has not been studied earlier. In winter depression, circadian rhythms have been described as both phase-delayed and poorly entrained to the 24-hour day (17), and patients with winter depression also seem to be more sensitive to melatonin suppression by bright or dim light than referents (18). Bright light also has mood-lifting effects on subjects with subsyndromal seasonal symptoms (19). The efficacy of light therapy may be partly explained by improvement in disturbed circadian rhythmicity. In this study, the light exposure was not aimed at producing circadian phase shifts, and the fact that neither circadian 
phase nor melatonin excretion was measured limits the interpretation of the results. We hypothesize that subjects with the seasonality trait may have preexisting circadian disturbances that render them more vulnerable to the detrimental effects of shift work, but possibly also are more sensitive to bright light stimuli. These results must however be interpreted with caution, as we also found that season (summer or winter) had no influence. This result is surprising, as the subjects with the seasonality trait would be expected to suffer more from season-related symptoms during wintertime. It may be that the abrupt change in the circadian rhythms related to night work is such a strong disturbing factor that season-related symptoms are disguised by it.

Shift work may be especially hazardous for older workers (20), and aging seems to decrease the ability to recover after several night shifts (21). Older age has been connected to increased "morningness" and slower circadian adjustment to shift work (21). In this study, however, the circadian type did not correlate with age, and age was not found to influence the effect, even after the cut-off point was raised from 40 to 45 or 50 years.

A shortcoming of the study was that all the subjects were women. This restriction limited the generalizability of the results, although there is no clear evidence of gender differences in the adaptation to night work (22). Another limitation is that the number of night shifts during the study periods varied between the subjects. This difference was unavoidable considering the study design. However, this variation was taken into account in the statistical analysis, and therefore it does not compromise the results.

The SSC mixes symptoms felt during and after the night shift. Thus it cannot be used to investigate circadian phase changes or physiological adaptation to changing work schedules. The questionnaire was chosen because the study focused on subjective complaints related to shift work, a condition resembling the external desynchronization of circadian rhythms in jet lag.

In conclusion, our results suggest that brief, timed exposure to bright light is an effective and well-tolerated means of alleviating night, work-induced subjective symptoms among women, especially those who routinely experience seasonal changes in mood and behavior.

\section{Acknowledgments}

We wish to express our gratitude to all the participants. Philips DAP, Espoo, Finland, provided the light boxes for research use in this study.

\section{References}

1. Czeisler CA, Moore-Ede MC, Coleman RM. Rotating shift work schedules that disrupt sleep are improved by applying circadian principles. Science 1982;217:460-3.

2. Eastman CI, Martin SK. How to use light and dark to produce circadian adaptation to night shift work. Ann Med 1999;3 1:87-98.

3. Partonen T, Lönnqvist J. Seasonal affective disorder. Lancet 1998;352:1369-74.

4. Partonen T, Lönnqvist J. Bright light improves vitality and alleviates distress in healthy people. J Affect Disord 2000; 57:55-61.

5. Costa G, Ghirlanda G, Minors DS, Waterhouse JM. Effect of bright light on tolerance to night work. Scand J Work Environ Health 1993;19:414-20.

6. Spitzer RL, Terman M, Williams JB, Terman JS, Malt UF, Singer F, et al. Jet lag: clinical features, validation of a new syndrome-specific scale, and lack of response to melatonin in a randomized, double-blind trial. Am J Psychiatry 1999;156: 1392-6.

7. Rosenthal NE, Genhart MJ, Sack DA, Skwerer RG, Wehr TA. Seasonal affective disorder and its relevance for the understanding and treatment of bulimia. In: Hudson JI, Pope HG, editors. The psychobiology of bulimia. Washington (DC): American Psychiatric Press; 1987. p 205-28.

8. Puska P, Tuomilehto J, Salonen J, Nissinen A, Virtamo J, Björkqvist $\mathrm{S}$, et al. Community control of cardiovascular diseases: the North Karelia project. Copenhagen: WHO Regional Office for Europe; 1981.

9. Hays RD, Sherbourne CD, Mazel RM. The RAND 36-item health survey 1.0. Health Econ 1993;2:217-27.

10. Horne JA, Ostberg O. A self-assessment questionnaire to determine morningness-eveningness in human circadian rhythms. Int J Chronobiol 1976;4:97-110.

11. Bartko JJ, Kasper S. Seasonal changes in mood and behavior: a cluster analytic approach. Psychiatry Res 1989;28:227-39.

12. Ware JE Jr, Sherbourne CD. The MOS 36-item short-form health survey (SF-36), I: conceptual framework and item selection. Med Care 1992;30:473-83.

13. Lindstrom MJ, Bates DM. Newton-Raphson and EM algorithms for linear mixed-effects models for repeated-measures data. J Am Stat Assoc 1988;83:1014-22.

14. Iwata $\mathrm{N}$, Ichii S, Egashira K. Effects of bright artificial light on subjective mood of shift work nurses. Ind Health 1997; 35:41-7.

15. Foret J, Daurat A, Tirilly G. Effect of bright light at night on core temperature, subjective alertness and performance as a function of exposure time. Scand J Work Environ Health 1998;24 suppl 3:115-20.

16. Campbell SS. Effects of timed bright-light exposure on shiftwork adaptation in middle-aged subjects. Sleep 1995;18: 408-16.

17. Teicher MH, Glod CA, Magnus E, Harper D, Benson G, Krueger K, et al. Circadian rest-activity disturbances in seasonal affective disorder. Arch Gen Psychiatry 1997;54:12430.

18. Thompson C, Stinson D, Smith A. Seasonal affective disorder and season-dependent abnormalities of melatonin suppression by light. Lancet 1990;336:703-6.

19. Kasper S, Rogers SL, Yancey A, Schulz PM, Skwerer RG, Rosenthal NE. Phototherapy in individuals with and without subsyndromal seasonal affective disorder. Arch Gen Psychiatry $1989 ; 46: 837-44$.

Scand J Work Environ Health 2003, vol 29, no 1 
20. Härmä MI, Ilmarinen JE. Towards the 24-hour society new approaches for aging shift workers? Scand J Work Environ Health 1999;25 (6, special issue):610-5.

21. Härmä MI, Hakola T, Åkerstedt T, Laitinen JT. Age and adjustment to night work. Occup Environ Med 1994;51:56873.
22. Hakola T, Härmä MI, Laitinen JT. Circadian adjustment of men and women to night work. Scand J Work Environ Health 1996;22:133-8.

Received for publication: 20 March 2002

\section{Appendix}

\section{Items on the Scale for Shiftwork Complaints}

How much have you been bothered by the following symptoms during or after this night shift? Scale of 0-4: $0=$ not at all, $1=$ a little bit, $2=$ moderately, $3=$ quite a bit, $4=$ extremely.

Decreased alertness before 0400

Decreased alertness after 0400

Sleepiness or falling asleep before 1000

Sleepiness or falling asleep after 1000

Fatigue or tiring easily

Physical clumsiness

Trouble concentrating or thinking clearly

Disorientation

Trouble with memory

General feeling of weakness

Light-headed, dizzy, or other uncomfortable sensations in the head Lethargy or sluggish feeling

Loss of appetite

Trouble falling asleep during the day

Craving for sweets

Feeling sad or low in spirit

Loss of pleasure or motivation 